\title{
Formation of Two-Dimensional Concentration Pulses on Microdesigned Composite Catalyst Surfaces
}

\author{
Michael Pollmann, Harm Hinrich Rotermund, and Gerhard Ertl \\ Fritz-Haber-Institut der Max-Planck-Gesellschaft, Faradayweg 4-6, 14195 Berlin, Germany \\ Xiujiang Li and Ioannis G. Kevrekidis \\ Department of Chemical Engineering, Princeton University, Princeton, New Jersey 08544
}

(Received 28 November 2000)

\begin{abstract}
We study the effect of microdesigned composite geometries on pattern formation during the catalytic oxidation of $\mathrm{CO}$ on Pt-Ti, Pt-Rh, and Pt-Pd composite catalysts. In particular, we find experimentally (and rationalize through modeling) that adsorbate surface transport through the second (active) component drastically affects the shapes and interactions of concentration patterns (traveling pulses) observed on pure Pt.
\end{abstract}

DOI: $10.1103 /$ PhysRevLett.86.6038

The use, over the last ten years, of spatially resolving techniques such as photoemission electron microscopy (PEEM) [1], and others [2,3], has allowed the real-time, in situ observation of spatiotemporal pattern formation on catalytic surfaces. These observations have important implications for the understanding of the transport and reaction mechanisms underlying catalytic reactions. At the same time, the generic features of the instabilities and bifurcations involved have made catalytic reactions a fruitful model medium for the study of such phenomena, leading to insights for pattern forming systems at large. A recent promising research avenue is the use of microfabrication techniques to construct microreacting domains with controlled shapes and sizes. The boundaries of these domains can be inert: in our case $2000 \AA$ "tall” Ti walls, presumably oxidizing to $\mathrm{TiO}_{2}$ under reaction conditions, which can be thought of as no-flux, inert, insulating boundaries. Shape, size, and dimensionality effects have been systematically studied on such microdesigned, inert boundary surfaces $[4,5]$. More recently, active boundaries are being constructed: the domains that surround the active Pt catalyst are no more inert, tall walls; they are, instead, covered by relatively thin layers $(50-300 \AA$ height $)$ of another catalyst (e.g., Pd, Rh) [6-8]. The motivation behind the fabrication of these composite catalysts is the desire to combine different catalytic activities through surface transport (modulated through geometrical design) to improve the overall catalyst activity/selectivity $[9,10]$.

In this paper we study a crucial intermediate step of this program: an active, pattern forming catalyst $[\mathrm{Pt}(110)$ in the excitable regime], surrounded by an active, nonpattern forming catalyst (in our case $\mathrm{Rh}$ or Pd). Related studies with a passive "just diffusion, no reaction" companion medium have recently been reported [11,12].

The evaporated, active, nonpattern forming catalyst effectively serves as an alternative supply for some of the reactants to the active, pattern forming one (the Pt). This additional source of reactants by surface diffusion through
PACS numbers: 82.40.Bj, 05.45.-a, 82.40.Np, 82.45.Jn

the interface boundary can drastically affect the shape, interactions, and speed of the patterns (pulses) on the Pt. The effect can be tuned through design of the composite geometry and the choice of its metal components.

We study CO oxidation in the 400-480 K temperature range for partial pressure of oxygen of $4 \times 10^{-4}$ mbar and for $\mathrm{CO}$ pressures in the $10^{-5}$ mbar range. The surfaces we employ are composites created by microlithographic deposition of a second metal (Ti, Rh, Pd) on a Pt(110) single crystal substrate; the patterned layers were typically $300 \AA$ in height (except for the $\mathrm{Ti}$, which was approximately $2000 \AA$ tall). The measurements are made using PEEM [1]. The sample is illuminated by a UV lamp; photoelectrons emitted from the surface are imaged via electron optics, intensified by a channel plate, and displayed on a fluorescent screen. The intensity distribution of this image is directly correlated with the local work function on the sample surface. The clean Pt(110) surface has the lowest work function and appears bright in the images. An O-covered $\operatorname{Pt}(110)$ surface appears dark because of the high dipole moment of $\mathrm{O}$ atoms, increasing the work function. The work function of a CO-covered surface lies in between, resulting in gray areas in PEEM images. After the crystal is brought in the PEEM-UHV chamber it is cleaned by repeated sputter/annealing cycles. To avoid intense alloying of the evaporated metal with the Pt substrate, the crystal was heated only to about $700 \mathrm{~K}$. Under comparable conditions in a different set of experiments the extent of alloying was found to be less than $2 \mu \mathrm{m}$ wide at the interface between two component areas of the composite (typically stripes, with widths greater than $10-20 \mu \mathrm{m}$ each) [9].

We model these shapes qualitatively, using a simplification of the established mechanistic $\mathrm{CO}$ oxidation model [13]. We use for the boundaries between the evaporated, active component and the $\mathrm{Pt}$ an approximate mixed (Robin) boundary condition (BC). The results are contrasted with those obtained with inert, no-flux $\left(\mathrm{TiO}_{2}\right)$ boundaries. 
Under our typical experimental conditions the Pt catalytic surface falls into the excitable regime, where pulses initiate and propagate upon a sufficient excitation [14]. We use a simplified, dimensionless two species model to describe the dynamics in this regime [15]:

$$
\begin{aligned}
u_{t} & =\nabla^{2} u-\frac{1}{\epsilon} u(u-1)\left(u-\frac{b+w}{a}\right), \\
w_{t} & =f(u)-w,
\end{aligned}
$$

where the time and space units correspond approximately to $2 \mathrm{~s}$ and $1.25 \mu \mathrm{m}$, respectively. The operating conditions $\left(P_{\mathrm{CO}}, P_{\mathrm{O}_{2}}\right.$, and surface temperature $\left.T\right)$ are embedded in the parameters $a, b$, and $\epsilon>0 . u$ is roughly $1-\theta_{\mathrm{CO}}$ and $w$ gives the degree of $1 \times 2$ surface reconstruction. The CO-covered stationary state corresponds to $(u, w)=(0,0)$ in the model, while the oxygen-covered state is represented by $(u, w)=(1,1) . f(u)$ is an experimental fit to the rate of change of the surface structure and takes the following form for a $\mathrm{Pt}(110)$ surface:

$$
f(u)= \begin{cases}0 & u<\frac{1}{3}, \\ 1-6.75 u(u-1)^{2} & \frac{1}{3} \leq u \leq 1, \\ 1 & u>1\end{cases}
$$

The length of the channel is much larger than the typical width of an oxygen pulse and periodic BCs are imposed at the two ends of the channel.

Under experimental conditions, the Rh (or Pd) part of the composite remains almost unchanged and appears uniform. We are thus able to approximate this problem of the two coupled domains for simulations with a reduced problem in a single domain (the Pt part) with appropriate BCs. Based on experimental observations we will assume that the $\mathrm{Rh}$ domains separating the Pt channels remain essentially uniformly covered by $\mathrm{CO}$. We model the flux of $\mathrm{CO}$ from these domains to the Pt using a mass transfer coefficient (a simplified, Robin, some times also called "albedo" $\mathrm{BC}$ ). The flux of $\mathrm{CO}$ normal to the stripe boundary (for an isotropic medium) is taken proportional to the difference in chemical potentials for $\mathrm{CO}$ across the interface; the chemical potential depends nonlinearly on the coverage, and by linearizing this difference we obtain

$$
\hat{\mathbf{n}} \cdot \frac{\partial u}{\partial y} \hat{\mathbf{y}}=-k(u-d) .
$$

Here $y$ is the direction across the channel (see Fig. 1), $u$ is related to the chemical potential of $\mathrm{CO}$ on the Pt side of the boundary, while $d$ is related to the chemical potential of $\mathrm{CO}$ on the (assumed CO-saturated) $\mathrm{Rh}$, far away from the interface. Normally one should solve the diffusion equation on the Rh, matching $\mathrm{CO}$ fluxes at the interface and with full nonlinear expressions for the chemical potential and its differences. We assume here a linear(ized) relationship between chemical potential and the surface coverage (so that $k$, our mass transfer coefficient in the form of a surface Sherwood number, is approximated by a constant). This assumption is, we believe, justified for a qualitative study.

In this work we concentrate on the most primitive pattern building blocks: pulse trains, and, in particular, quasione-dimensional $\mathrm{O}$ pulses propagating in narrow linear $\mathrm{Pt}$ channels surrounded by "second active metal" (Rh, Pd) stripes. The reference case of an inactive Ti overlayer can be theoretically described by no-flux boundaries $(k=0)$ [5]. In this case, both the experiments and the simulations exhibit $\mathrm{O}$ pulses with a rectangular, essentially onedimensional (flat in the $y$ direction) shape (Fig. 1). The figure shows the experimental image, the numerical $u$-field solution, and the $u, w$ profiles along the $x$ direction of $\mathrm{O}$ pulses in a channel with inert, no-flux boundaries.

Figure 2a shows measurements with $\mathrm{Rh} / \mathrm{Pt}$ (a) and $\mathrm{Pd} / \mathrm{Pt}$ samples (c) (for the latter see [7]). The pulses are clearly no longer rectangular. In the $\mathrm{Rh} / \mathrm{Pt}$ case they exhibit "triangular" shapes. The (gray-scale contour level) front is only slightly bent inwards at the sides, but the back is drastically constricted - it forms a sharp tail. The pulses for the $\mathrm{Pd} / \mathrm{Pt}$ sample exhibit a circular or oval shape. They appear more symmetric with respect to the shape of their front and back. To simulate these cases we facilitate transport from the $\mathrm{CO}$ reservoir (slightly raise the $k$ value).

One may expect that $\mathrm{CO}$ diffusing through the interface will react with the oxygen in the channel and thus "eat up" the oxygen pulse close to the boundary. Because of the asymmetry between the front and the back of the pulse, more "erosion" will occur at the back side. As shown in Fig. 1, the $1 \times 2(w)$ reconstruction percentage is higher at the back than it is at the front. Since the sticking coefficient of oxygen on a $1 \times 2$ structure is about $50 \%$ smaller (a)

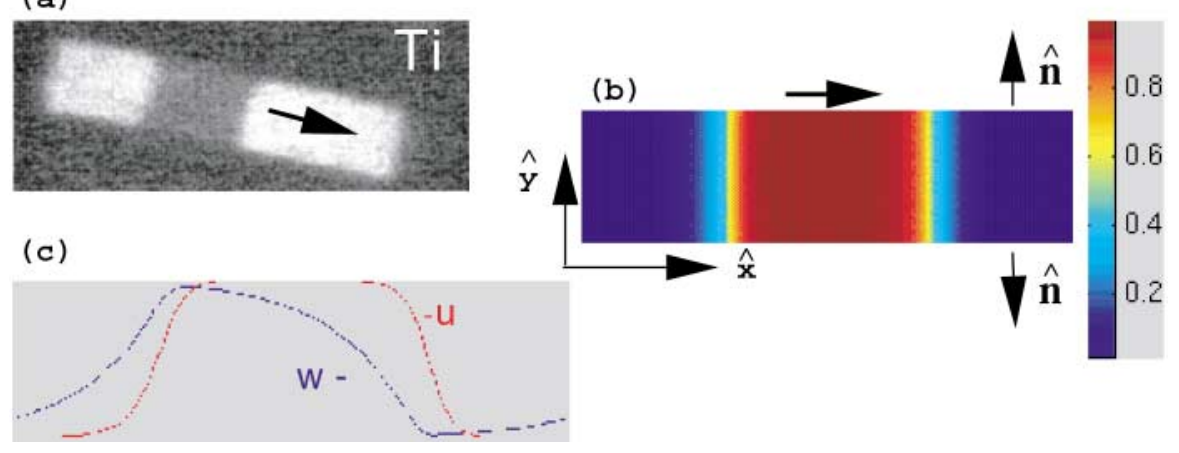

FIG. 1 (color). (a) Experimental PEEM image of an $\mathrm{O}$ pulse in a $20 \mu \mathrm{m}$ wide $\mathrm{Pt}$ channel on a $\mathrm{Ti} / \mathrm{Pt}$ surface $(T=$ $390 \mathrm{~K}, \quad P_{\mathrm{O}_{2}}=4 \times 10^{-4}$ mbar, $P_{\mathrm{CO}}=$ $4.1 \times 10^{-5}$ mbar). (b),(c) $2 \mathrm{D}$ pulse simulation: $u$-field solution and $u, w$ profiles along the $x$ direction at $k=0(a=0.84$, $b=-0.0509, \epsilon=0.0784$ ). The red color corresponds to higher concentrations of $u$ (around 1); the blue color is associated with the state $u=0$. The same color scheme as well as the simulation box size ( $L=20, W=6$ ) applies to all subsequent figures. 
(a)

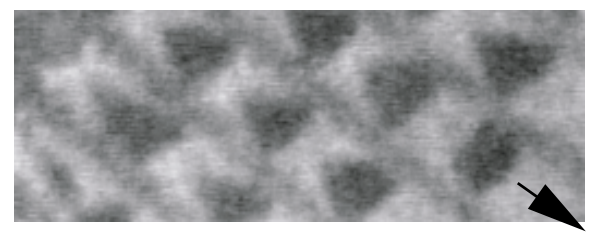

(b)

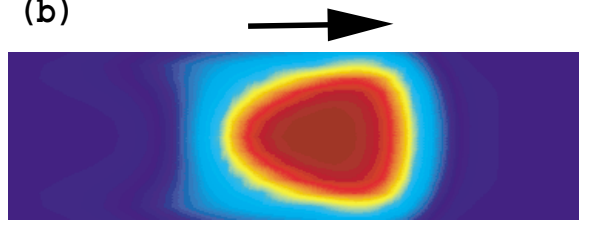

(c)

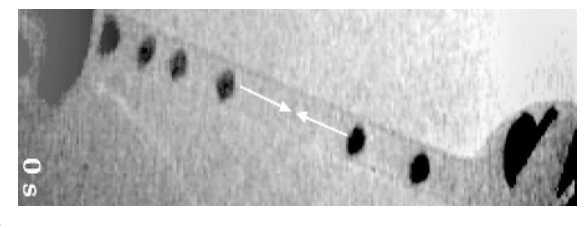

(d)

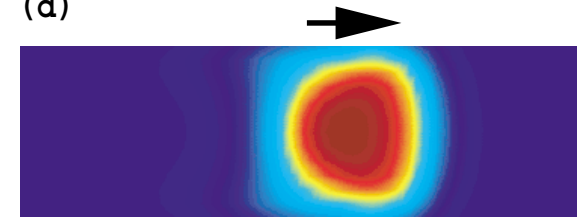

FIG. 2 (color). (a): Experimental PEEM images of $\mathrm{O}$ pulses in $10 \mu \mathrm{m}$ wide $\mathrm{Pt}$ channels during $\mathrm{CO}$ oxidation on a $\mathrm{Rh} / \mathrm{Pt}$ surface $\left(T=440 \mathrm{~K}, P_{\mathrm{O}_{2}}=4 \times 10^{-4} \mathrm{mbar}\right.$, $P_{\mathrm{CO}}=3.4 \times 10^{-5} \mathrm{mbar}$ ). (b) The oxygen pulse at $k=1.8148, d=0, a=$ $0.84, b=-0.0509, \epsilon=0.0784$. (c) Experimental PEEM image of $\mathrm{O}$ pulses in $20 \mu \mathrm{m}$ wide $\mathrm{Pt}$ channels on a $\mathrm{Pd} / \mathrm{Pt}$ surface $\left(T=425 \mathrm{~K}, P_{\mathrm{O}_{2}}=4 \times 10^{-4} \mathrm{mbar}\right.$, $P_{\mathrm{CO}}=3.8 \times 10^{-5} \mathrm{mbar}$ ). (d) The oxygen pulse at $k=1.8148, d=-0.1693$, $a=0.84, b=-0.0509$, and $\epsilon=0.0784$. than it is on a $1 \times 1$ structure, it is conceivable that the oxygen is more sensitive to the invasion of $\mathrm{CO}$ at the back of the pulse. The pulse has a distinct triangular shape when its back begins to detach from the boundary (Fig. 2b). When the transport of $\mathrm{CO}$ from the reservoir is even larger, both the front and the back of the pulse strongly feel this CO-mediated oxygen erosion. The pulse detaches from the boundary completely and exhibits an almost round shape, as can be seen in the PEEM image and in the qualitative numerical simulations reproduced in Figs. $2 c$ and $2 d$. Here, Pd plays for Pt the role of an even "richer" (compared to $\mathrm{Rh}) \mathrm{CO}$ reservoir. In the numerical simulation, the $\mathrm{CO}$ flux through the side was increased by approximately $50 \%$.

In Fig. 2a we observe the fact that the triangular shape of the $\mathrm{O}$ pulses for the $\mathrm{Rh} / \mathrm{Pt}$ system is not perfectly symmetric with respect to the channel centerline. This misalignment occurs due to the anisotropy of the reconstructed Pt(110)-surface structure: a slow and a fast diffusion axis exist. This anisotropy may affect the orientation of the triangular pulse depending on the angle of the microfabricated channels with respect to the underlying substrate orientation. Figure 3 a shows an example with channels which are practically aligned (in contrast to the slightly more misaligned Fig. 2a). In this case the triangles are essentially symmetric with respect to the channel centerline.

It is well known that, on extended $\mathrm{Pt}(110)$-single crystal surfaces, intrinsic defects act as pattern initiation or pinning centers; in a similar spirit, the $\mathrm{Rh} / \mathrm{Pt}$ interface acts as a "defect" causing localized front initiation (e.g., [6]). Figure 3a shows an array of isolated Pt channels in a "sea" of $\mathrm{Rh}$ : the channel end acts as a nucleation source for $\mathrm{O}$ pulses. The "strength" of this defect determines the frequency of the pulse initiation; in particular, regions of high boundary curvature (the corners) consistently act as pulse initiation locations (see Fig. 3b). This is consistent with qualitative theoretical predictions (e.g., [9]); anisotropy obviously favors one of the two corners. It is interesting to observe the transient evolution of the interpulse distances for the wave trains initiated there upon a parameter change. When this frequency is high enough, only the first initiated pulse propagates freely; the subsequent ones interact with it and among themselves. These interactions may ultimately lead to "gluing" of successively initiated pulses (Fig. 3d).

Figure 4 shows another example of pulse interaction in a channel. In this case the pulses exhibit a "boomerang" structure: their fronts are bent backwards. This behavior is probably due to the refractory time of the Pt reconstruction (the "inhibitor" in the model). In between the passage of $\mathrm{O}$ pulses the Pt-surface structure has to undergo the transition from $1 \times 1$ to $1 \times 2$ and back to $1 \times 1$ (associated with a change from a $\mathrm{CO}$-covered to an O-covered surface and back to $\mathrm{CO}$ covered again). Depending on the speed of and spacing between the pulses (and the relation to this refractory time) the front shape of a pulse may be affected by the shape of the back of the forerunner pulse.

Figure 3c shows a final observation of a two-pulse unit. The first pulse is normal, whereas the latter is much smaller and strongly asymmetric. We have observed in many instances such "mother-child" pulse systems to propagate stably without a shape change. Pulses with twodimensional structures occasionally reminiscent of ours, but in a different experiment (annular gel reactor fed from both sides, "excyclons") have been studied, along with their interactions, by Dulos et al. [16].

We studied pattern formation on catalyst composites, and, in particular, the effect of the composite on the shape and interaction of pulses on the $\mathrm{Pt}(110)$ part of the composite. Pulses constitute the main building blocks of more complex patterns, such as targets and spirals. We believe that our composites provide a new reactant source mechanism for the $\mathrm{Pt}$ component of the composite: reactant adsorption on the companion component, and transport laterally, through surface diffusion, to the Pt. This "perturbation" to the pattern forming dynamics on the Pt can be tailored through the choice of a companion component, as well as through the geometry of the composite (composite composition, stripe width). We found that the qualitative effect on the shape and interaction of the pulses (the introduction of distinct two-dimensional features based on the front-back asymmetry of the pulse) was well captured theoretically through a mechanistic model of $\mathrm{CO}$ oxidation. The model was also capable of qualitatively confirming speed trends with parameters such as accelerations 

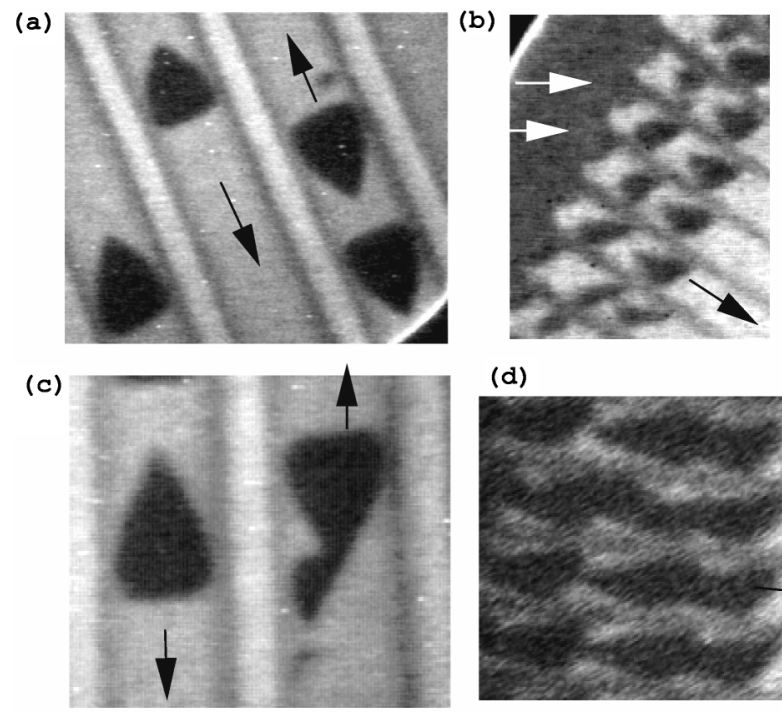

(d)

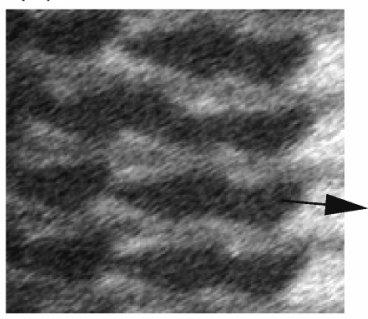

FIG. 3. Experimental PEEM image of $\mathrm{O}$ pulses in $22 \mu \mathrm{m}$ wide $\mathrm{Pt}$ channels on a $\mathrm{Rh} / \mathrm{Pt}$ surface $\left(T=440 \mathrm{~K}, P_{\mathrm{O}_{2}}=4 \times\right.$ $10^{-4}$ mbar, $P_{\mathrm{CO}}=3.2 \times 10^{-5}$ mbar). The pulses are symmetric with respect to the channel centerline. (b) Pulse initiation at the corners (high curvature regions) of the channel "inlet," marked by white arrows (see text). Conditions as in Fig. 2a. (c) Instance of "mother and child" asymmetric pulse pairs. (d) Pulse interactions leading to "gluing" ( $T=436 \mathrm{~K}$, $\left.P_{\mathrm{O}_{2}}=4 \times 10^{-4} \mathrm{mbar}, P_{\mathrm{CO}}=2.6 \times 10^{-5} \mathrm{mbar}\right)$.

with temperature and stripe width. More quantitative studies (with the companion medium entering directly and not just as a BC) are underway, and preliminary results show the possibility of dynamic instabilities in the composite medium. We are also studying quantitatively the effects of anisotropy, temperature, and the coupling of pulse trains in adjacent channels through surface diffusion across narrow
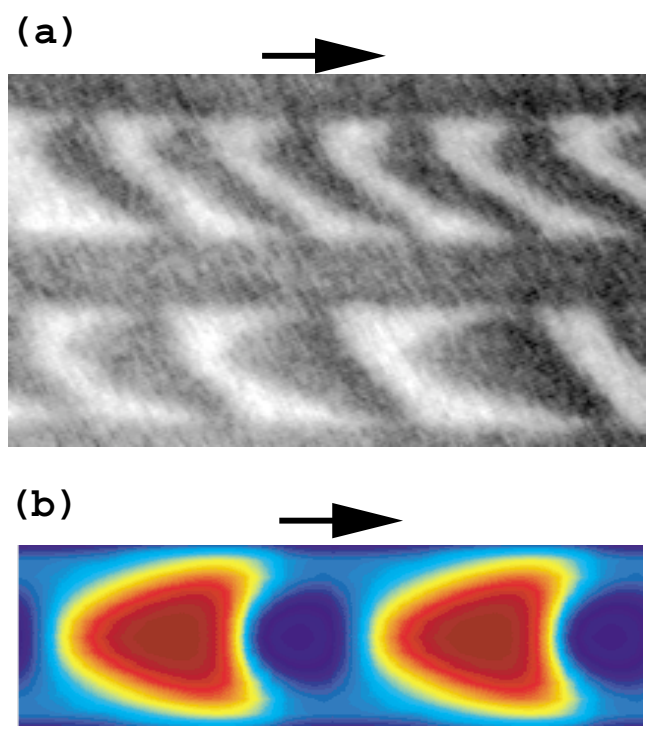

FIG. 4 (color). (a) Experimental PEEM image of $\mathrm{O}$ pulses in $20 \mu \mathrm{m}$ wide $\mathrm{Pt}$ channels on a $\mathrm{Pt} / \mathrm{Rh}$ surface at $T=440 \mathrm{~K}$, $P_{\mathrm{O}_{2}}=4 \times 10^{-4}$ mbar, $P_{\mathrm{CO}}=3.4 \times 10^{-5}$ mbar. (b) The computed oxygen pulse at $k=1.8148, d=0, a=0.885$, $b=-0.0509$, and $\epsilon=0.0784$. companion corridors (a phenomenon discussed by Steinbock et al. [17]). An additional experimental direction involves the fabrication of a "spatiotemporally varying composite": a Pt(110) surface addressable through a narrow laser beam, capable of writing "higher temperature" stripes on the Pt [18].

We gratefully acknowledge the partial support of the DFG through SFB 555 "Complex nonlinear systems" (M.P., H. H.R.) and of NSF, AFOSR, and the Humboldt Foundation (X. L., I. G. K.). Preliminary computations using the "Virtual Laboratory" of Professor A. Mikhailov (M.P.) and the generous computational assistance of Dr. S. Shvartsman are also acknowledged.

[1] H. H. Rotermund, W. Engel, M. Kordesch, and G. Ertl, Nature (London) 343, 355 (1990).

[2] E. Bauer, Rep. Prog. Phys. 57, 895 (1994).

[3] H. H. Rotermund, G. Haas, R. U. Franz, R. M. Tromp, and G. Ertl, Science 270, 608 (1995).

[4] M.D. Graham, I. G. Kevrekidis, K. Asakura, J. Lauterbach, K. Krischer, H. H. Rotermund, and G. Ertl, Science 264, 80 (1994); M. D. Graham, M. Bär, I. G. Kevrekidis, K. Asakura, J. Lauterbach, H. H. Rotermund, and G. Ertl, Phys. Rev. E 52, 76 (1995); G. Haas, M. Bär, I. G. Kevrekidis, P. B. Rasmussen, H. H. Rotermund, and G. Ertl, Phys. Rev. Lett. 75, 3560 (1995); E. Schütz, N. Hartmann, I. G. Kevrekidis, and R. Imbihl, Catal. Lett. 54, 181 (1998); N. Hartmann, I. G. Kevrekidis, and R. Imbihl, J. Chem. Phys. 112, 6795 (2000).

[5] M. Bär, A. K. Bangia, I. G. Kevrekidis, G. Haas, H. H. Rotermund, and G. Ertl, J. Phys. Chem. 100, 19106 (1996).

[6] M. Bär, I. G. Kevrekidis, H.H. Rotermund, and G. Ertl, Phys. Rev. E 52, R5739 (1995).

[7] J. Lauterbach, K. Asakura, P. B. Rasmussen, H. H. Rotermund, M. Bär, M.D. Graham, I. G. Kevrekidis, and G. Ertl, Physica (Amsterdam) 123D, 493 (1998).

[8] K. Agladze, A. Toth, T. Ichino, and K. Yoshikawa, J. Phys. Chem. A 104, 6677 (2000).

[9] S. Y. Shvartsman, E. Schütz, R. Imbihl, and I. G. Kevrekidis, Phys. Rev. Lett. 83, 2857 (1999).

[10] M. X. Yang, D. H. Gracias, P. W. Jacobs, and G. Somorjai, Langmuir 14, 1458 (1998).

[11] O. Steinbock, P. Kettunen, and K. Showalter, Science 269, 1857 (1995).

[12] K. Suzuki, T. Yoshinobu, and H. Iwasaki, J. Phys. Chem. A 104, 5154 (2000); K. Suzuki, T. Yoshinobu, and H. Iwasaki, J. Phys. Chem. A 104, 6602 (2000).

[13] K. Krischer, M. Eiswirth, and G. Ertl, J. Chem. Phys. 96, 9161 (1992).

[14] J. D. Murray, Mathematical Biology (Springer, New York, 1989).

[15] M. Bär, N. Gottschalk, M. Eiswirth, and G. Ertl, J. Chem. Phys. 100, 1202 (1994).

[16] E. Dulos, J. Boissonade, and P. DeKepper, Physica (Amsterdam) 188A, 120 (1992).

[17] O. Steinbock, P. Kettunen, and K. Showalter, J. Phys. Chem. 100, 18970 (1996).

[18] J. Wolff, I. G. Kevrekidis, and H.H. Rotermund (to be published). 